ARTICLE

https://doi.org/10.1038/s41467-019-11639-2

\title{
Horse immunization with short-chain consensus $\alpha$-neurotoxin generates antibodies against broad spectrum of elapid venomous species
}

Guillermo de la Rosa (1) ${ }^{1,2}$, Felipe Olvera ${ }^{1}$, Irving G. Archundia ${ }^{1}$, Bruno Lomonte $\mathbb{B}^{3}{ }^{3}$, Alejandro Alagón ${ }^{1} \&$ Gerardo Corzo (1) ${ }^{1}$

Antivenoms are fundamental in the therapy for snakebites. In elapid venoms, there are toxins, e.g. short-chain $\alpha$-neurotoxins, which are quite abundant, highly toxic, and consequently play a major role in envenomation processes. The core problem is that such $\alpha$-neurotoxins are weakly immunogenic, and many current elapid antivenoms show low reactivity towards them. We have previously developed a recombinant consensus short-chain $\alpha$-neurotoxin (ScNtx) based on sequences from the most lethal elapid venoms from America, Africa, Asia, and Oceania. Here we report that an antivenom generated by immunizing horses with ScNtx can successfully neutralize the lethality of pure recombinant and native short-chain $\alpha$-neurotoxins, as well as whole neurotoxic elapid venoms from diverse genera such as Micrurus, Dendroaspis, Naja, Walterinnesia, Ophiophagus and Hydrophis. These results provide a proof-ofprinciple for using recombinant proteins with rationally designed consensus sequences as universal immunogens for developing next-generation antivenoms with higher effectiveness and broader neutralizing capacity.

\footnotetext{
${ }^{1}$ Departamento de Medicina Molecular y Bioprocesos, Instituto de Biotecnología, Universidad Nacional Autónoma de México - UNAM, Apartado Postal 510-3, Cuernavaca Morelos 61500, Mexico. ${ }^{2}$ The Donnelly Centre for Cellular and Biomolecular Research, University of Toronto, Toronto, ON M5S3E1, Canada. ${ }^{3}$ Instituto Clodomiro Picado, Universidad de Costa Rica, San José 11501, Costa Rica. Correspondence and requests for materials should be addressed to G.d.I R. (email: guillermo.delarosa.h@gmail.com) or to G.C. (email: corzo@ibt.unam.mx)
} 
S nakebite is one the most neglected diseases, especially in the poorest tropical countries near the Equator. Literature analysis based on statistical estimates shows that up to 5.5 million snakebites could occur every year, yielding to more than 100,000 deaths worldwide ${ }^{1}$. The only venom-specific life-saving treatment proved as effective and recommended by the World Health Organization (WHO) is the timely parenteral application of snake antivenom ${ }^{2}$. Essentially, the active principle of antivenoms is a polyclonal mixture of immunoglobulins, or fragments thereof, like Fab or $\mathrm{F}\left(\mathrm{ab}^{\prime}\right)_{2}$. These are typically derived from the sera of hyper-immunized animals, mainly horses, and are able to neutralize the different venom toxins to prevent their deleterious effects ${ }^{3}$.

In antivenom design and production, venoms from snakes responsible for causing high morbidity and mortality are commonly selected as immunogens ${ }^{3}$. In compliance with the host immune system, both toxic and non-toxic venom components elicit an antibody response; as a result, antivenoms contain collections of antibodies against both relevant and non-relevant components, which can affect antivenom efficacy. Postsynaptic $\alpha-$ neurotoxins are one of the main toxic elements in elapid venoms and the most poorly recognized components by current antivenoms ${ }^{4-6}$, despite being rather abundant protein components in venoms used as immunogens.

$\alpha$-Neurotoxins are classified as type I (short-chain), type II (long-chain), and non-conventional neurotoxins ${ }^{7}$. Short-chain $\alpha$ neurotoxins (60-62 amino acids) have been associated with the high toxicity of many elapid venoms. They bind to the nicotinic acetylcholine receptors (nAChR) blocking neurotransmitter binding. Accordingly, they cause non-depolarizing blockade and consequently abolish neurotransmission, resembling curaremimetic effects ${ }^{8}$. Under an elapid snakebite scenario, therefore, an effective anti-elapid therapy should have a collection of IgGs, F $\left(\mathrm{ab}^{\prime}\right)_{2}$, or Fab fragments able to properly neutralize $\alpha$-neurotoxins in order to prevent or reverse postsynaptic neurotoxicity caused by these curare-mimetic toxins ${ }^{9}$.

Aiming to develop complementary strategies to improve the antibody response and cross-recognition towards short-chain $\alpha$ neurotoxins, our previous work focused on the design, recombinant expression, and biochemical characterization of a consensus type I a-neurotoxin with generic traits, here called $\mathrm{ScNtx}^{10}$. In this study, the ScNtx is used as an immunogen in horses, which are the preferred animal used for production of snake antivenoms

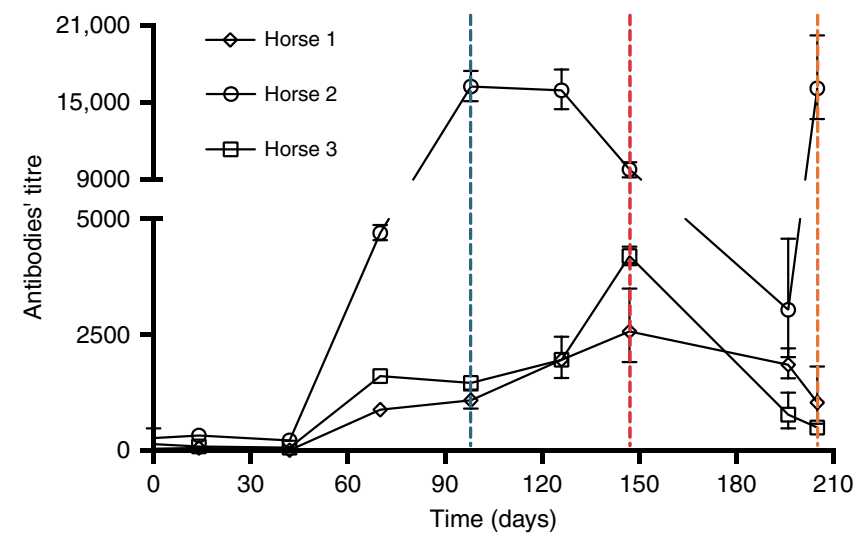

Fig. 1 Time course of the specific antibody response of horses against the consensus short-chain $\alpha$-neurotoxin ( $\mathrm{ScNtx}$ ). Horse hyperimmune sera raised against the ScNtx were titrated by enzyme-linked immunosorbent assay (ELISA). Dashed lines indicate samples used for neutralization tests, corresponding to days 98 (blue), 147 (red), and 205 (orange) of the immunization. Points represent mean \pm SD of triplicate wells of the ELISA available on the market worldwide. The resulting anti-ScNtx experimental antivenom (EAv) efficacy and species coverage, expressed as median effective dose $\left(\mathrm{ED}_{50}\right)$, are systematically evaluated. Thus, our goal is to determine the extent of protection provided by this antivenom in mouse lethality tests against the challenge of isolated recombinant type I neurotoxins, as a proof of concept, and also against whole elapid venoms from snakes considered of highest medical importance in the Americas, Africa, Asia, and Oceania. Our results strongly suggest that a consensus $a$-neurotoxin as a rational-based immunogen in the production of antivenoms against neurotoxic elapid venoms could result in a product with a wide spectrum of specificity, efficacy, and affordability.

\section{Results}

ScNtx as immunogen. In order to better understand the role of type I $\alpha$-neurotoxins within the overall lethality of whole elapid venoms, we developed a horse-derived antivenom using a biologically active type I consensus $\alpha$-neurotoxin, ScNtx, as a "universal" immunogen. The ScNtx was designed to show better antigenic properties (high therapeutic antibody titers) and thus to produce better antivenoms. We found that the group of three horses, immunized in a multi-site manner with increasing $\mathrm{ScNtx}$ doses (from 10 to $400 \mu \mathrm{g}$ ), produced antibodies that recognized the homologous immunogen in enzyme-linked immunosorbent assay (ELISA). Serum analysis shows that the response was rising over 210 days, meaning that increasing doses of ScNtx were efficient in inducing antibody production in these large animals. The titer of antibodies had a tendency to increase in response to repeated injections of the neurotoxin; nonetheless, ScNtx evoked a different response in the three animals (Fig. 1), one of them reaching a titer as high as 18,000 .

Lethal potencies. The efficient assessment of antivenoms $\left(\mathrm{ED}_{50}\right)$ is based on their ability to neutralize the lethal effect of snake venoms (lethal dose, $\left.50 \%\left(\mathrm{LD}_{50}\right)\right)$. Therefore, we first determined the $\mathrm{LD}_{50}$ for all toxins and venoms studied in this work (Table 1). In total, we used 4 short-chain $\alpha$-neurotoxins and 29 elapid snake venoms from species distributed in different world regions. The short-chain $\alpha$-neurotoxins had intravenous (IV) $\mathrm{LD}_{50} \mathrm{~s}$ ranging from 1.2 to $19.0 \mu \mathrm{g} /$ mouse. Concerning the elapid venoms, those from the Micrurus genus had $\mathrm{LD}_{50} \mathrm{~s}$ ranging from $3.8 \mu \mathrm{g} / \mathrm{mouse}$ (Micrurus browni) to $15.0 \mu \mathrm{g} / \mathrm{mouse}$ (Micrurus tener tener); venoms from Dendroaspis ranged from 5.3 to $17.0 \mu \mathrm{g} / \mathrm{mouse}$; the $\mathrm{LD}_{50} \mathrm{~s}$ of the venoms from Naja genus were from 0.9 (Naja haje from Morocco) to $22.7 \mu \mathrm{g} /$ mouse (Naja mossambica); venoms from Pseudechis were from 6.9 to $8.9 \mu \mathrm{g} /$ mouse, and finally, the $\mathrm{LD}_{50} \mathrm{~s}$ of venoms from Ophiophagus hannah, Walterinnesia aegyptia, Oxyuranus scutellatus, and sea snake Hydrophis (Pelamis) platura were $11.5,4.9,0.7$, and $3.9 \mu \mathrm{g} /$ mouse, respectively.

ScNtx elicits neutralizing antisera. First, we assessed the individual response and maturation of the immune response based on the neutralization potency of three serum samples collected throughout the immunization period of the horses, corresponding to days 98, 147, and 206. Results presented in Fig. 2 revealed that animals exhibited differences in neutralization potency against $a$-neurotoxins. In general, effective doses $\left(\mathrm{ED}_{50}\right)$ were higher in samples from day 98 and lower from day 206, indicating an increase in neutralizing potency over time.

Second, we developed two lots of EAvs called Lot \#1 and Lot \#2. Lot \#1 contains antibodies from sera pooled from horse 2 at days 147 and 205, and Lot \#2 has antibodies from the day 708 . Both lots have a protein content of $50 \mathrm{mg} / \mathrm{mL}$. Lot \#1 was 
Table 1 Median LD $_{50}$ and ED 50 values of $\alpha$-neurotoxins or elapid venoms, as well as median values of antivenom (EAv)

\begin{tabular}{|c|c|c|c|c|c|}
\hline $\begin{array}{l}\alpha \text {-Neurotoxin/ } \\
\text { venom }\end{array}$ & $\begin{array}{l}\text { LD }_{50} \text { in } \mu \mathrm{g} / \mathrm{mice} \\
(95 \% \mathrm{Cl})\end{array}$ & $\begin{array}{l}\text { Horse EAv ED } \\
(\mu \mathrm{L} / \text { mice })(95 \% \\
\mathrm{CI}) \text {, Lot \#1 }\end{array}$ & $\begin{array}{l}\text { Horse EAv ED } 50 \\
\text { ( } \mu \mathrm{L} / \mathrm{mice})(95 \% \\
\text { CI), Lot \#2 }\end{array}$ & $\begin{array}{l}\text { Average } \mathrm{mg} \text { toxin } \\
\text { or venom } / \mathrm{mL} \text { EAv }\end{array}$ & $\begin{array}{l}\text { Average } L_{50} \text { of } \\
\text { toxin or venom/ } \\
\text { mL EAv }\end{array}$ \\
\hline \multicolumn{6}{|l|}{$\alpha$-Neurotoxins } \\
\hline ScNtx & $3.9(3.8-3.9)$ & $49.1(47.8-50.4)$ & $40-45$ & 0.23 & 61.1 \\
\hline MlatA1 & $19(18-20)$ & $45.4(44.1-46.8)$ & ND & 1.25 & 66.1 \\
\hline r.D.H & $3(2.8-3.2)$ & $31.7(31.7-31.8)$ & ND & 0.28 & 94.6 \\
\hline P01424 & $1.2(1.1-1.3)$ & $34.3(33.7-34.9)$ & ND & 0.17 & 145.7 \\
\hline \multicolumn{6}{|l|}{ Venoms } \\
\hline Micrurus browni & $3.8(3.5-4.1)$ & NN & ND & - & - \\
\hline Micrurus diastema & $6(5.5-6.5)$ & 191.5 (188.7-194.3) & NN & 0.09 & 15.6 \\
\hline Micrurus distans & $12(10-14)$ & NN & ND & - & - \\
\hline Micrurus fulvius & $4(3.8-4.2)$ & NN & ND & - & - \\
\hline $\begin{array}{l}\text { Micrurus } \\
\text { laticorallis }\end{array}$ & $10(9.1-11.4)$ & $171.1(166.0-175.0)$ & NN & 0.17 & 17.5 \\
\hline $\begin{array}{l}\text { Micrurus } \\
\text { nigrocinctus }\end{array}$ & $7(5-9)$ & $56.5(54.8-58.1)$ & NN & 0.37 & 53.1 \\
\hline $\begin{array}{l}\text { Micrurus } \\
\text { surinamensis }\end{array}$ & $10(9.5-10.5)$ & $51.2(49.7-52.7)$ & $25.6(20.0-30.0)$ & 0.78 & 78.1 \\
\hline $\begin{array}{l}\text { Micrurus tener } \\
\text { tener }\end{array}$ & $15(13-17)$ & NN & ND & - & - \\
\hline $\begin{array}{l}\text { Dendroaspis } \\
\text { angusticeps }\end{array}$ & $17(16-18)$ & $178.0(171.3-185.4)$ & NN & 0.28 & 16.8 \\
\hline $\begin{array}{l}\text { Dendroaspis } \\
\text { polylepis }\end{array}$ & $5.3(5-5.5)$ & $149.1(141.2-157.3)$ & NN & 0.16 & 20.1 \\
\hline Dendroaspis viridis & $12(11.9-12.2)$ & NN & ND & - & - \\
\hline Naja atra & $8(7.7-8.2)$ & $37.5(35.5-39.5)$ & $34.2(28.5-41.1)$ & 0.67 & 83.0 \\
\hline Naja haje ${ }^{a}$ & $0.9(0.8-1)$ & $77.4(76.6-78.2)$ & $72.9(63.9-81.0)$ & 0.04 & 40.0 \\
\hline Naja kaouthia & $3.9(3.7-4.2)$ & $50.0(47.0-53.0)$ & $>400$ & 0.23 & 60.0 \\
\hline Naja katiensis & $20.5(18.4-22.7)$ & ND & $>400$ & - & - \\
\hline Naja oxiana & $8(7.0-9.8)$ & ND & $24.1(20.1-28.5)$ & $0.99^{b}$ & $124.4^{b}$ \\
\hline Naja melanoleuca & $6.5(6.4-6.6)$ & $72.3(69.9-74.7)$ & $306.0(301.0-311.0)$ & 0.11 & 15.8 \\
\hline Naja mossambica & $22.7(19.5-25.8)$ & NN & NN & - & - \\
\hline Naja naja naja & $9.4(9.3-9.6)$ & $65.5(63.1-67.7)$ & ND & 0.43 & 45.8 \\
\hline Naja nigricollis & $18(17-19)$ & NN & ND & - & - \\
\hline Naja nivea & $8.2(8-8.4)$ & $25(20-30)$ & $106.0(92.5-121.5)$ & 0.38 & 46.7 \\
\hline Naja nubiae & $8.3(8.1-8.4)$ & $173.7(169.1-178.4)$ & ND & 0.14 & 17.3 \\
\hline Naja pallida & $17(17.8-17.2)$ & NN & ND & - & - \\
\hline $\begin{array}{l}\text { Ophiophagus } \\
\text { hannah }\end{array}$ & $11.5(10.9-12.2)$ & $47.9(44.1-52.1)$ & $74.9(73.2-76.7)$ & 0.56 & 48.8 \\
\hline Hydrophis platura & $3.9(0.8-6.4)^{c}$ & $40-66$ & ND & No $<0.17$ & No $<45.4$ \\
\hline $\begin{array}{l}\text { Walterinnesia } \\
\text { aegyptia }\end{array}$ & $4.9(3.9-5.8)$ & $55.7(53.1-58.5)$ & $44.5(40.0-49.5)$ & 0.29 & 59.8 \\
\hline $\begin{array}{l}\text { Oxyuranus } \\
\text { scutellatus }\end{array}$ & $0.7(0.6-0.8)$ & ND & NN & - & - \\
\hline $\begin{array}{l}\text { Pseudechis } \\
\text { australis }\end{array}$ & $6.9(5.6-8.6)$ & ND & NN & - & - \\
\hline Pseudechis colleti & $8.9(8.4-9.5)$ & ND & NN & - & - \\
\hline
\end{tabular}

comprehensively evaluated, while Lot \#2 was used only used for some venoms.

ScNtx antivenom. The vast array of pathophysiological symptoms exhibited by elapid envenomation are related to presynaptic- and postsynaptic-acting toxins. Venomics and conventional research have unveiled many predominant presynaptic and predominant postsynaptic elapid venoms. Thus, we aimed to assess the extent of protection of our EAv against shortchain $\alpha$-neurotoxins and well-known elapid venoms from the Americas, Africa, Asia, and Oceania.
Efficacy of ScNtx antivenom against purified a-neurotoxins. The EAv Lot \#1 neutralized the lethal effect of purified type I aneurotoxins. The $\mathrm{ED}_{50}$ values determined for this preparation, against $\alpha$-neurotoxins, are expressed in $\mu \mathrm{L} /$ mouse and listed in Table 1. This anti-ScNtx preparation neutralized the lethality of all the purified $\alpha$-neurotoxins, with $\mathrm{ED}_{50}$ values around $40 \mu \mathrm{L} /$ mouse. EAv had an $\mathrm{ED}_{50}=49.1 \mu \mathrm{L} /$ mouse against the $\mathrm{ScNtx}$, meaning $230 \mu \mathrm{g}$ of ScNtx neutralized by $1 \mathrm{~mL} / \mathrm{EAv}$ or $230 \mu \mathrm{g}$ of toxin/50 mg of immunoglobulins. Lot \#2 had a similar range of potency. Similarly, as shown in Table 1 , EAv Lot \#1 had an $\mathrm{ED}_{50}$ $=45.1 \mu \mathrm{L} /$ mouse $(1.2 \mathrm{mg} / \mathrm{mL} \mathrm{EAv})$ and $\mathrm{ED}_{50}=31.7 \mu \mathrm{L} / \mathrm{mouse}$ $(280 \mu \mathrm{g} / \mathrm{mL}$ EAv) against recombinant MlatA1 and r.D.H, 


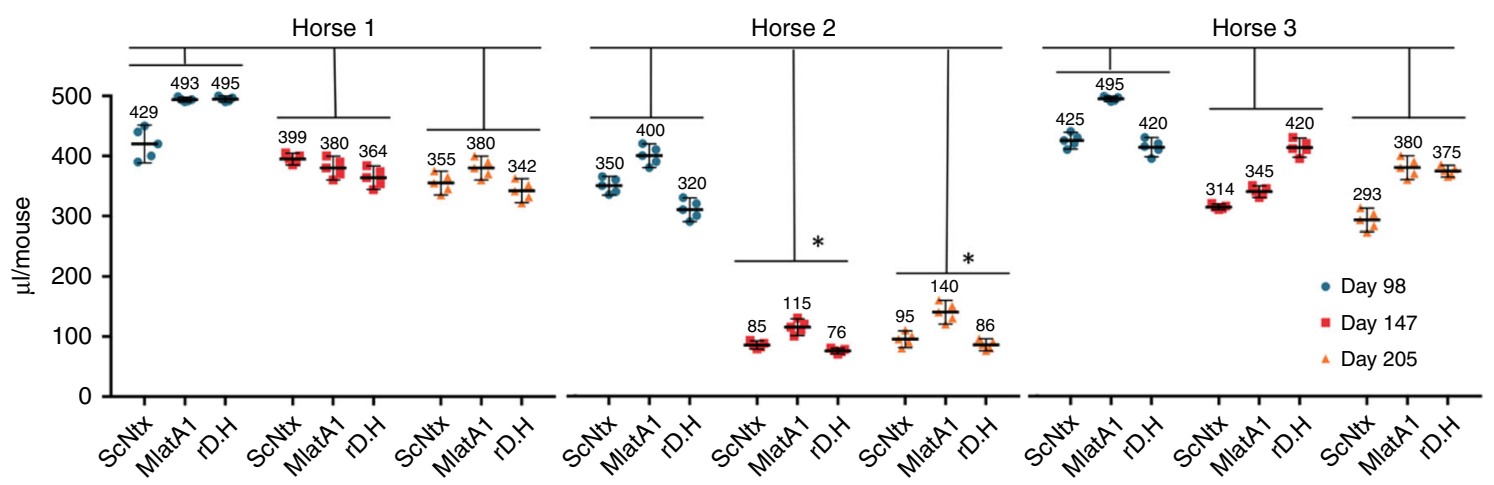

Fig. 2 Neutralization potencies, plotted as median effective dose $\left(E D_{50}\right)$. Serum samples were obtained from individual horses immunized with the consensus short-chain $\alpha$-neurotoxin (ScNtx) at days 98, 147, and 205. ED 50 values are expressed in $\mu \mathrm{L} / \mathrm{mouse}$ (volume of anti-ScNtx able to neutralize $3 \times$ $\mathrm{LD}_{50}$ (lethal dose, $50 \%$ ) of purified short-chain neurotoxins ScNtx, MlatA1 or rD.H.). Values were estimated in groups of five mice, injected by the intravenous route. Error bars represent the $95 \%$ confidence limits for the ED 50 value. The data obtained from the groups of "Horse" and "Day" of immunization were analyzed statistically by two-way analysis of variance (ANOVA) followed by paired Student's $t$ test. A $p$ value of $<0.05$ was considered significant, as indicated by *. Horse 2 produces antibodies with the best neutralization potencies from after the 147th day of immunization

\begin{tabular}{clll}
\hline Toxin & Amino acid sequence & $\begin{array}{c}\text { Identity } \\
\text { (\%) }\end{array}$ & $\begin{array}{c}\text { Accession } \\
\#\end{array}$ \\
\hline ScNtx & MICYNQQSSQPPTTKTCS--ETSCYKKTWRDHRGTIIERGCGCPKVKPGIKLHCCRTDKCNN & 100 & \\
rD.H & MICHNQQSSQPPTTKTCS--EGQCYKKTWRDHRGTIIERGCGCPTVKPGIHISCCASDKCNA & 85 & P86420 \\
MlatA1 & RICYNQQSSQPPTTKTCS--EGQCYKKTWRDHRGTIIERGCACPNVKPGIQISCCTSDKCNG & 84 & K9MCH1 \\
P01426 & LECHNQQSSQPPTTKTCP-GETNCYKKVWRDHRGTIIERGCGCPTVKPGIKLNCCTTDKCN & 83 & P01426 \\
P80548 & MICHNQQSSQPPTIKTCS--EGQCYKKTWRDHRGTISERGCGCPTVKPGIHISCCASDKCNA & 81 & P80548 \\
Atratoxin & LECHNQQSSQTPTTKTCS-GETNCYKKWWSDHRGTIIERGCGCPKVKPGVNLNCCTTDRCNN & 79 & AAR33036 \\
MS1 & MICYNQQSTEPPTTKTCS--EGQCYKKTWSDHRGTIIERGCACPNVKPGKISCCSSDKC & 79 & P86095 \\
P01424 & MECHNQQSSQPPTTKTCP-GETNCYKKQWSDHRGTIIERGCGCPSVKKGVKINCCTTDRCNN & 77 & P01424 \\
Pelamitoxin & MTCCNQQSSQPKTTNCA--ESSCYKKTWSDHRGTRIERGCGCPQVKSGIKLECCHTNECNN & 75 & P62388 \\
W-III & FVCHNQQSSQPPTTTNCSGGENKCYKKQWSDHRGSITERGCGCPTVKKGIKLHCCTTEKCNN & 73 & C1IC47 \\
\hline
\end{tabular}

Fig. 3 Multiple sequence alignment of the most lethal short-chain $\alpha$-neurotoxins. ScNtx: Synthetic consensus $\alpha$-neurotoxin ${ }^{10}$; rD.H: Micrurus diastema (recombinant)53; MlatA1: Micrurus laticollaris (recombinant)52; P01426: Naja spp.; P80548: Micrurus nigrocinctus4; Atratoxin: Naja atra; MS1: Micrurus surinamensis; P01424: Naja melanoleuca; pelamitoxin: Hydrophis platura; W-III: Walterinnesia aegyptia. Differences from the ScNtx are indicated by bold letters; cysteines and disulfide bridge arrangements are colored in blue

respectively, and an $\mathrm{ED}_{50}=34.3 \mu \mathrm{L} /$ mouse $(170 \mu \mathrm{g} / \mathrm{mL} \mathrm{EAv})$ against the native type I $\alpha$-neurotoxin, P01424.

Efficacy of ScNtx antivenom against coral snake venoms. Micrurus fulvius, M. tener, M. browni, and M. distans whole venoms are not neutralized by the EAv (efficient against type I $\alpha$-neurotoxins). On the other hand, EAv Lot \#1 neutralized M. laticollaris $\left(\mathrm{ED}_{50}=171.0 \mu \mathrm{L} / \mathrm{mouse}\right)$ and $M$. diastema $\left(\mathrm{ED}_{50}=191.0 \mu \mathrm{L} /\right.$ mouse) venoms with higher doses. That means that 170 and $90 \mu \mathrm{g}$ of each venom is neutralized by $1 \mathrm{~mL}$ of EAv $(50 \mathrm{mg})$. Nonetheless, venoms from these species but obtained from different individuals were not neutralized by Lot \#2. Similar findings were determined for the Central American coral snake Micrurus nigrocinctus. On the one hand, EAv Lot \#1 was able to successfully neutralize its lethality $\left(\mathrm{ED}_{50}=56.6 \mu \mathrm{L} /\right.$ mouse $)$. On the other hand, EAv Lot $\# 2$ did not neutralize the lethality of a different individual. Finally, for $M$. surinamensis both lots of EAv were highly efficient in neutralizing the lethality of the venom from the same individual snake $\left(\mathrm{ED}_{50}=\right.$ 51.6 and $25.6 \mu \mathrm{L} /$ mouse, respectively) (Table 1 ).

Efficacy of ScNtx antivenom against cobra venoms. To determine the efficacy of the EAv on more complex elapid venoms, we performed neutralization assays on 13 different species of spitting and non-spitting cobras. In our findings, the lethality of venoms of the spitting cobras Naja nigricollis, N. pallida, N. katiensis, and
N. mossambica was not abolished by the EAv, unlike the $N$. nubiae venom $\left(\mathrm{ED}_{50}=173 \mu \mathrm{L} /\right.$ mouse) (Table 1$)$. We next addressed whether the EAv was effective against the non-spitting cobras. As a first finding, our data indicated that non-spitting cobras (Naja) show, in most cases, the most lethal venoms. We also found that they are the best neutralized by the EAv here characterized. For example, the $N$. haje venom used in this work had a $\mathrm{LD}_{50}=0.9 \mu \mathrm{g} /$ mouse, and its lethality was inhibited by the EAv Lot $\# 1\left(\mathrm{ED}_{50}=77.4 \mu \mathrm{L} /\right.$ mouse $)$. Similar efficacy was found for the venoms from $N$. melanoleuca $\left(\mathrm{ED}_{50}=72.4 \mu \mathrm{L} /\right.$ mouse $)$ and N. naja $\left(\mathrm{ED}_{50}=65.5 \mu \mathrm{L} /\right.$ mouse $)$.

\section{Discussion}

Snake venoms are an exquisite panoply of molecules, mostly proteins, contributing differentially in the pathophysiological effects in snakebites. Nonetheless, such complexity is expressed as a relatively small but important set of clinical manifestations, such as flaccid paralysis, local tissue damage, systemic myolysis, hemorrhage, and coagulation alterations, among others. For efficient immunotherapy against snakebite envenoming, proper neutralization of toxins playing important roles is required ${ }^{11}$. A key set of potential targets are the elapid a-neurotoxins, which cause flaccid paralysis leading to death by respiratory failure. However, one of the main drawbacks related to $a$-neurotoxins is their poor immunogenicity. Additionally, many works have 
suggested the role of type I a-neurotoxins in the lethal potency of venoms stemming from a-neurotoxins' high toxicity, molecular action, and abundance ${ }^{5,12}$.

Overall, the efficacy of the experimental antivenom ( $\mathrm{ScNtx}$ antivenom) correlates with the presence of type I $\alpha$-neurotoxins and some other members of the 3FTx family within the venoms. First, the neutralization of pure $\alpha$-neurotoxins by the EAv suggest shared immunogenic determinants among these toxins. In fact, comparing their amino acid sequences, shown in Fig. 3, we qualitatively deduced a high conservation in two regions: from the first to the third cysteine (first loop) and from the second to the fourth cysteine (Cys) residue, which bears the region corresponding to the second loop. The latter, particularly, contains the amino acid residues involved in the toxin-receptor interaction. As expected, the conservation of some residues at the second loop among the ScNtx sequence and other 3FTxs such as fasciculins ${ }^{13}$ and long-chain neurotoxins ${ }^{13-15}$ agrees with the conclusion of some researchers that postulates this region as a conserved neutralization epitope on $\alpha$-neurotoxins ${ }^{16}$. Henceforth, in-depth studies on mapping the interactions of ScNtx with its elicited horse antibodies would be revealing to this hypothesis.

Micrurus produces potent neurotoxic venoms that act on the neuromuscular junction. It has been demonstrated that there are two main protein families among them: phospholipases $\mathrm{A}_{2}$ $\left(\mathrm{PLA}_{2} \mathrm{~s}\right)$ and three-finger toxins (3FTxs). In a global view, the proportion of $\mathrm{PLA}_{2} \mathrm{~s}$ vs. 3FTxs varies among North American and South American species ${ }^{17}$. For instance, venoms from $M$. fulvius (Florida) and $M$. tener (Texas) are $\mathrm{PLA}_{2}$ predominant, while $M$. surinamensis (South America) contains a 3FTx-rich venom almost devoid of $\mathrm{PLA}_{2}$ activity. For the former, it has been demonstrated that such $\mathrm{PLA}_{2} \mathrm{~s}$ are the main lethal components in the mouse model of envenomation ${ }^{18}$. In the case of $M$. nigrocinctus (Central America), which contains a venom that is $\mathrm{PLA}_{2}$ predominant over $3 \mathrm{FTxs}^{4}$, EAv was able to successfully neutralize its lethality $\left(\mathrm{ED}_{50}=56.6 \mu \mathrm{L} /\right.$ mouse $)$ and had similar efficacy to some commercial antivenoms ranging from 63 to $123 \mu \mathrm{L} /$ mouse (Coralmyn $^{\circledast}$ and anti-Micrurus, respectively) ${ }^{19}$. We conclude that the $\alpha$-neurotoxin fraction is highly relevant for its lethality. In this case such effect could be led by the main short-chain $\alpha$ neurotoxin P80548, which presents $81 \%$ identity compared to the $\mathrm{ScNtx}$, as shown in Fig. 3, which is also the most lethal toxin in this venom ${ }^{4}$. Similarly, both the EAv Lot \#1 and Lot \#2 were highly efficient in neutralizing the lethality of $M$. surinamensis (3FTx-rich venom) venom, which contains a large arsenal of $\alpha-$ neurotoxins that shares high identity in sequence to $\mathrm{ScNtx}(>74 \%$, Fig. 3$)^{20}$.

The EAv is efficient against 3FTx-rich cobra (Naja) venoms. Cobra venoms contain 3FTxs, $\mathrm{PLA}_{2} \mathrm{~s}$, metalloproteinases (SVMPs), Cys-rich secretory proteins, and L-amino oxidases

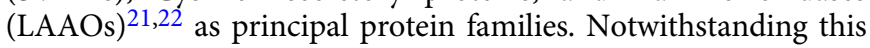
set of toxins, most human envenomations result in neurological affectations and in some cases serious local injuries, which correlate with the predominance of 3FTxs, SVMPs, or PLA ${ }_{2}$ s. For instance, N. nigricollis, N. katiensis, N. pallida, and N. mossambica are rich in cytotoxins/cardiotoxins, primarily, followed by $\mathrm{PLA}_{2} \mathrm{~S}$ and metalloproteinases. These toxins cause local tissue necrosis and hemorrhage; therefore, the inefficacy of our EAv on these venoms agrees with their proteomics and distinctive clinical manifestation $^{22,23}$. On the other hand, the EAv does neutralize 3FTx-predominant cobra venoms such as $N$. nubiae (type I neurotoxin content $\left.\sim 12.6 \%^{22}\right), N$. haje $(3 \mathrm{FTx}=\sim 60 \%)^{24,25}, \mathrm{~N}$. melanoleuca $(3 \mathrm{FTx}=\sim 57 \%)^{25,26}$-one possible target could be the $\alpha$-neurotoxin $P 01424\left(\mathrm{LD}_{50}=0.9 \mu \mathrm{g} /\right.$ mouse $)$ that greatly influences its lethality and that we showed is neutralized by the $\mathrm{EAv}-N$. naja $(3 \mathrm{FTx}=\sim 63.8 \%)^{27}$ - which is one of the main species responsible for snakebite mortality in South-Asia-and
Naja oxiana (type I neurotoxin content $>12 \%)^{28}$. The present study has only investigated the neutralization of the venom/toxininduced lethality. Consequently, this work clearly has some limitations. The most important lies in the fact that we cannot demonstrate whether EAv cross-reacts with other 3FTxs, such as type II, and cytotoxin/cardiotoxins. However, our results indicate that cross-reaction might occur. For instance, antibodies to ScNtx in the EAv were able to protect from lethal doses of the venoms from $N$. atra, N. nivea, and N. kaouthia $(3 \mathrm{FTx}=56-78 \%)$, which are not only rich in cytotoxins (e.g., CTX) ${ }^{29-31}$ but also in type II a-neurotoxins (e.g., a-cobratoxin P0139) ${ }^{21-32}$. It is well known that $N$. atra venom produces cytotoxicity resulting from the action of the group of toxins historically known as CTX ${ }^{29,31}$, and likewise produces neurotoxicity caused mainly by type I $\alpha$ neurotoxins (e.g., atratoxins) and muscarinic toxins (MTs), which are also considered to play important roles in the envenoming process. Naja nivea venom, in turn, displays important myotoxic, cardiotoxic, and neurotoxic activities ${ }^{31}$. These results reveal as a whole the specific role of type I a-neurotoxins vs. other toxic elements on the lethality of these venoms and an arguable crossreactivity among other 3FTxs.

To test the role of a likely cross-reactivity among 3FTxs, we used the venom of Mambas. They produce venoms that contain a rich cocktail of rapid-acting neurotoxins ${ }^{32}$. Human envenomation by mambas can lead to neurotoxicity with no reports of local tissue damage ${ }^{8}$. Toxins acting on potassium channels (Kv), L-type calcium channels, acid-sensing ion channels ${ }^{33}$, cholinergic receptors $^{34}$, and others acting on platelet aggregation ${ }^{35}$ have been reported. Nonetheless, the evidence suggests that 3FTxs, such as MTs, fasciculins, and $\alpha$-neurotoxins, coupled with presynapticacting Kunitz-type proteinase inhibitor-like (dendrotoxins) could be highly relevant for mamba venoms lethality ${ }^{36,37}$. Under an anti- $\alpha$-neurotoxin view, neutralization of these ScNtxs was achieved for Dendroaspis angusticeps and D. polylepis, but $D$. viridis lethal effect remained unaltered. These results show how important the set of diverse neurotoxins (e.g., MTs, fasciculins, dendrotoxins, long-chain a-neurotoxins) are to the lethality of Dendroaspis and some Naja venoms. They also suggest that the neutralization of the a-neurotoxins, and the possible cross-reactivity, is not sufficient to provide protection since mice, even when they survived, in all cases showed envenomation symptoms.

Here, it is worth mentioning that long- and short-chain $a$ neurotoxins are equally important during elapid envenomation, and more studies are needed to decipher the pharmacokinetics and biochemistry of each one of these neurotoxins. Also, the use of mice as an animal model for interpreting neutralization of venoms that are rich in short- or long-chain $\alpha$-neurotoxins has to be revised. Silva et al. ${ }^{38}$ found differential susceptibility of human and rat nAChR to short-chain a-neurotoxins, but not to longchain $\alpha$-neurotoxins. This finding may represent an important challenge for the current assessment of the preclinical efficacy of antivenoms in mouse models.

Hence, further studies should take into consideration the possible combined action among mamba toxins in order to postulate additional antigens for immunization. Moreover, our results promote further research in order to have a deep insight about cross-reactivity and the variation in terms of efficacy between the different lots of EAvs against Dendroaspis venoms. In this regard, it is important to underline that such variation was not observed for all venoms. For instance, for the same batch of king cobra venom (Ophiophagus hannah) and black snake (Walterinnesia aegyptia) venom the $\mathrm{ED}_{50} \mathrm{~s}$ among EAv lots were similar. We know that these venoms are 3FTx predominant; nonetheless, their complexity is markedly different. King cobra venom, for example, contains LAAOs, SVMPs, and PLA $\mathrm{s}^{39}$. Among the rich repertoire of 3FTxs, it has been proven that long- 
chain $\alpha$-neurotoxins play a key role in its lethality given the fact that specific antibodies produced against them attenuate the venom toxicity ${ }^{40,41}$. Importantly, other toxins, like the shortchain a-neurotoxins, even though they are less abundant, are substantially crucial for the venom lethality ${ }^{42}$. Our results support this observation, as we demonstrated that $O$. hannah lethality can be efficiently abolished by both lots of EAv, possibly by acting on both long-chain and short-chain neurotoxins. On the other hand, $W$. aegyptia venom, while less complex, is very active postsynaptically, causing life-threatening neurotoxic envenomation ${ }^{43}$. The most abundant toxins in this venom are short-chain $\alpha$ neurotoxins, $\mathrm{PLA}_{2} \mathrm{~s}$, and Kunitz-type proteinase inhibitor-like proteins ${ }^{43,44}$. Nevertheless, only short-chain $\alpha$-neurotoxins have been considered as targets in the development of a new antiwalterinnesia antivenom. For instance, the short-chain a-neurotoxin $\mathrm{T}_{\mathrm{III}}$, which is the most abundant toxin in the venom, was used to develop an antivenom that neutralizes $50 \mathrm{LD}_{50} / \mathrm{mL}^{44}$; comparing this, $1 \mathrm{~mL}$ of EAv neutralizes $53 \mathrm{LD}_{50 \text { s }}$, supporting the concept that $W$. aegyptia lethality is driven by its principal components, namely short-chained a-neurotoxins. Similarly, the most abundant and lethal component of the sea snake $H$. platura $(3 \mathrm{FTx}=\sim 50 \%)$ venom is the $a$-neurotoxin known as pelamitoxin (P62388) and its proteoforms ${ }^{45}$. Owing to their high identity to the $\mathrm{ScNtx}$, both $\mathrm{T}_{\mathrm{III}}$ and pelamitoxin are likely the targets of the neutralizing anti-ScNtx antibodies.

To question whether the EAv could neutralize or attenuate the lethality of elapid venoms from Oceania, we used the venom of two genera distributed in Australia and parts of New Guinea: Pseudechis australis, P. colleti, and Oxyuranus scutellatus. These venoms contain short-chain neurotoxins and they are also active at the presynaptic level like some Micrurus venoms ${ }^{46}$. Nonetheless, when tested, EAv did not have any effect on their lethality. Although these venoms cause neurotoxicity that leads to respiratory arrest, our result suggest that this might be caused, mainly, by other group of neurotoxins such as long-chain neurotoxins or presynaptically acting $\mathrm{PLA}_{2} \mathrm{~S}$ rather than by shortchain neurotoxins. Alternatively, these venoms could contain short-chain neurotoxins that are more antigenically divergent from the consensus sequence immunogen here studied.

In conclusion, in this paper we have described the range of efficacy for a polyspecific horse-derived experimental antivenom capable of neutralizing medically important elapid venoms distributed throughout the Americas, Africa, Middle East, and some regions of Asia. The evidence from this study has revealed the importance of short-chain a-neurotoxins in the lethality of several Micrurus, Naja, Dendroaspis, Walterinnesia, Ophiophagus, Oxyuranus, Pseudechis, and Hydrophis venoms.

Furthermore, due to the fact that snake venoms can present intra-species variability in their composition, recombinant proteins could be a constant source of active toxins (immunogens) for better antidotes. For this, it is clear that further work needs to be done to elucidate more medically relevant toxins. Correspondingly, it is important for future research to consider variation in venoms composition to understand how this variability could also influence both envenomation signs and antivenom efficacy 47,48 . The rational concept of using a recombinant neurotoxin with "universal" consensus sequence to produce a broad-specificity anti-elapid antivenom provides the framework for a strategy to develop either animal-derived or recombinant antivenoms. We hope that our research will be useful in solving the difficulty of conceiving alternative immunogens to create better therapeutic antivenoms with higher neutralizing potencies and broader coverages.

\footnotetext{
Methods

Venoms and toxins. Venoms used in this study were from different sources.
} Certified, $N$. melanoleuca (715.030 and 307.150), N. katiensis (code not provided),
N. oxiana (911.040), N. pallida (316.000), N. nivea (524.010), N. nubiae (101.030) N. mossambica (505.010), N. nigricollis (105.030), N. haje (822.090), N. kaouthia (506.000), N. atra (920.100), D. polylepis (218.020), D. angusticeps (305.00), D. viridis (815.050), W. aegyptia (729.030), and O. hannah (923.090) venoms were from Latoxan (Valence, France); N. nivea (NNC019) was from SA Venom Suppliers (Louis Trichardt, South Africa); N. naja naja (4NN9001) from Ventoxin; the australian venoms Oxyuranus scutellatus (OSS0319EAS, Cooktown, Australia), P. australis (PA0319EAS, Coen Cape York, Australia), P. colleti (PC0319EAS, Vergemont, Australia) were acquired through "La Nauyaca" (UMA INE/CITES/ DFYFS-HERP-E-0003-MOR/98, Morelos, Mexico); venoms from M. fulvius (08.31.10) and M. tener tener (02.16.09) were from National Natural Toxins Research Center; and venoms from M. laticollaris (two individuals), M. browni, $M$. diastema (two individuals), $M$. distans, $M$. nigrocinctus (two individuals), $M$. surinamensis, and sea snake $H$. (Pelamis) platura ${ }^{24}$ were obtained manually by milking multiple adult snakes at the "Instituto de Biotecnología" (UNAM, Mexico, permit SGPA/DGVS/010526/18) and "Instituto Clodomiro Picado" (UCR, Costa Rica, permit CICUA-021-17) serpentariums.

Expression and purification of $\boldsymbol{\alpha}$-neurotoxins. Stored $\mathrm{pQE} 30 / \mathrm{ScNtx}^{10}, \mathrm{pQE} 30 /$ MlatA $1^{49}$, and pQE30/r.D. $\mathrm{H}^{50}$ plasmids were transformed into Escherichia coli K12-derived Origami cells for expression following the same conditions as in our previous work (for a more detailed description see de la Rosa et al. ${ }^{10}$ ). In short, expressed recombinant ScNtx, MlatA1 or r.D.H were submitted to a two-step purification process: by Ni-NTA (Ni-nitrilotriacetic acid) affinity chromatography according to the method of polyhistidine-tagged proteins user manual (Qiagen); and by reversed-phase high-performance liquid chromatography (RP-HPLC) (Agilent 1100 series; Agilent, CA) loading them onto an analytical $\mathrm{C}_{18}$ column $\left(4.6 \times 250 \mathrm{~mm}^{2}, \mathrm{VYDAC}^{\circledast}\right)$. Elution was carried out at $1 \mathrm{~mL} / \mathrm{min}$ for $60 \mathrm{~min}$ by applying a gradient starting at $10 \%$ of an aqueous acetonitrile solution $/ 0.1 \%$ trifluoroacetic acid (TFA) and ending at $60 \%$. Similarly, in order to isolate the $\alpha$ neurotoxin P014242 (UniProt access code) from N. melanoleuca, $2 \mathrm{mg}$ of crude venom were fractionated by RP-HPLC using a gradient toward acetonitrile solution $/ 0.1 \%$ TFA maintaining $0 \% 5 \mathrm{~min}, 0-15 \%$ over $10 \mathrm{~min}, 15-45 \%$ over $60 \mathrm{~min}$, $45-70 \%$ over $10 \mathrm{~min}$, and $70-80$ over $5 \mathrm{~min}^{31}$. Finally, all identities were confirmed by mass spectrometry.

Animals. Mice strain CD-1 (18-20 g) were purchased from Harlan Mexico and kept at animal facilities at the "Instituto de Biotecnología", Mexico, and also, they were provided and kept at animal facility at the "Instituto Clodomiro Picado", Costa Rica. Adult male horses (400-500 kg) were from Ranch "Ojo de Agua" (Puebla, Mexico). All animals received regular veterinary supervision and were maintained under good conditions and controlled environments. They received water and food ad libitum. Proper animal handling, in order to minimize distress and discomfort, was always conducted towards maximizing the animal welfare during experimentation according to Mexican legislation for the use of laboratory animals (Norma Oficial Mexicana, 1999, NOM-062-ZOO-1999). Additionally, internal animal handling was according to the Animal Care and Bioethics Committee at the "Instituto de Biotecnología" (ethical approval CB/IBt/Project \# 254) and "Instituto Clodomiro Picado" (ethical approval code: CICUA-021-17), which supervised and approved all animal experiments.

Immunization, antisera collection, and sera fractionation. Three horses were immunized by multi-site intradermal and subcutaneous route ${ }^{51}$, starting with $5 \mu \mathrm{g}$ and ending with $1000 \mu \mathrm{g}$ of ScNtx per horse. The first immunization of $5 \mu \mathrm{g}$ was performed in $0.5 \mathrm{~mL}$ phosphate-buffered saline plus $0.7 \mathrm{~mL}$ incomplete Freund's adjuvant (IFA) by intradermal route. Horses were boosted 14, 28, 42, 56, 70, 84, 98, $112,126,140$, and 196 days later with $5,5,10,20,40,80,150,300,300,500,500$, and $1000 \mu \mathrm{g}$ of the ScNtx, alternating IFA and Alum. No adjuvant was used on the seventh and the last two immunizations. For all cases, horses were bled at weekly intervals until day 147 and the last two blood samples were taken at days 196 and 205. Then, after a 488-day hiatus, only horse 2 was boosted four times at weekly intervals with 300 (day 684), 500 (day 692), 1000 (day 700), and $1800 \mu \mathrm{g}$ (day 708). IFA and Alum were used in these first two boosts and no adjuvants were used in the last two; a blood sample was taken 1 week after the last immunization. Animals' blood was allowed to clot at $37^{\circ} \mathrm{C}$ for at least $2 \mathrm{~h}$, chilled on ice for $1 \mathrm{~h}$, and centrifuged at $4000 \times g$. Serum was collected and stored frozen at $-20^{\circ} \mathrm{C}$ until used.

Finally, horse immunoglobulins were obtained by caprylic (octanoic) acid method $^{52}$ described and recommended in the WHO guidelines for the production, control, and regulation of antivenoms ${ }^{53}$. Two different lots were prepared: Lot \#1 contains immunoglobulins from days 147 and 205, and Lot \#2 from day 708. Each Lot has a protein content of $50 \mathrm{mg} / \mathrm{mL}$.

Median $\mathbf{L D}_{\mathbf{5 0}}$ and $\mathbf{E D}_{\mathbf{5 0}}$. Lethal potencies for toxins and whole venoms (in $\mu \mathrm{g}$ dry weight per mouse) were determined by calculating the $\mathrm{LD}_{50}$, which is defined as the amount of venom that produces the death of $50 \%$ of the mice challenged. Briefly, five mice per group were injected by IV route and the $\mathrm{LD}_{50}$ was obtained from the plot analysis of mice mortality (at $24 \mathrm{~h}$ after injection) vs. toxin or venom dose used. The $\mathrm{LD}_{50}$ was expressed in $\mu \mathrm{g} /$ mouse. For neutralization experiments, $3 \times$ 
$\mathrm{LD}_{50}$ of either toxin or whole venom were pre-incubated $30 \mathrm{~min}$ at $37^{\circ} \mathrm{C}$ with varying volumes of antiserum and then injected by IV route. After $24 \mathrm{~h}$, median effective dose $\left(\mathrm{ED}_{50}\right)$ was calculated from the plot of survival percent (at $48 \mathrm{~h}$ ) vs. antiserum dose, and it is defined as the volume of antiserum able to protect $50 \%$ of the mice challenged (five mice per group) ${ }^{19}$. Only for the native type I $\alpha$ neurotoxin P014242, N. haje and Oxyuranus scutellatus, due to their high toxicity, $5 \times \mathrm{LD}_{50}$ of each were used for $\mathrm{ED}_{50}$ calculation. Control groups tested without antiserum or with pre-immune horse sera always resulted in $100 \%$ mortality; likewise, $400 \mu \mathrm{L}(50 \mathrm{mg} / \mathrm{mL})$ of purified horse pre-immune immunoglobulins showed no alterations in the envenomation process and mortality. The $\mathrm{ED}_{50}$ was expressed in microliters. Prism (GraphPad Inc., San Diego, CA) was used to calculate the data by non-linear regression. Experiments were carried out following the guidelines published by WHO involved in the production, control, and regulation of venoms and antivenoms ${ }^{54}$.

Antiserum titration. Each horse hyperimmune serum was tested by ELISA for the presence of specific antibodies against the ScNtx. Flat- bottom, 96-MicroWell ${ }^{\mathrm{sm}}$ polystyrene microtiter plates (Maxisorp Nunc) were coated with $100 \mu \mathrm{L} /$ well of $5 \mu \mathrm{g} / \mathrm{mL} \mathrm{ScNtx}$ in carbonate/bicarbonate stock solution at $\mathrm{pH} 9.5$ and incubated overnight at $4{ }^{\circ} \mathrm{C}$. After incubation, plates were rinsed three times with $200 \mu \mathrm{L} /$ well of rinsing buffer ( $50 \mathrm{mM}$ Tris- $\mathrm{HCl}, 150 \mathrm{mM} \mathrm{NaCl}, 0.05 \%$ Tween-20, and $\mathrm{pH} 8$ ). After that, it was added $150 \mu \mathrm{L} /$ well of blocking buffer $(50 \mathrm{mM}$ Tris- $\mathrm{HCl}, 5 \mathrm{mg} / \mathrm{mL}$ gelatin, $0.2 \%$ Tween-20, and $\mathrm{pH} 8$ ) and incubated $2 \mathrm{~h}$ at $37^{\circ} \mathrm{C}$. After a second rinsing cycle, serum anti-ScNtx (1:30 dilution) was mixed with vehicle buffer (50 mM Tris- $\mathrm{HCl}, 0.5 \mathrm{M} \mathrm{NaCl}, 1 \mathrm{mg} / \mathrm{mL}$ gelatin, $0.05 \%$ Tween-20, and $\mathrm{pH} 8$ ), and placed in the first well $(200 \mu \mathrm{L})$ and further serially diluted 1:3 (with the same buffer of the ELISA plates) from wells 2 to 11 , while well 12 contained only vehicle buffer. Plates were incubated for $1 \mathrm{~h}$ at $37^{\circ} \mathrm{C}$, and after rinsing twice; plates were incubated with $100 \mu \mathrm{L} /$ well of peroxidase-conjugated rabbit anti-horse immunoglobulins $\left(5 \times 10^{-4} \mu \mathrm{g} / \mathrm{mL}\right.$, Rockland) for $1 \mathrm{~h}$ at $37^{\circ} \mathrm{C}$. Plates were then rinsed three times with rinsing buffer and, finally, $100 \mu \mathrm{L} /$ well of peroxidase chromogenic substrate (soluble BM Blue POD substrate, Roche) were added, and incubated for $10 \mathrm{~min}$ at room temperature, in darkness. At the end of the incubation, the reaction was stopped with $100 \mu \mathrm{L} /$ well sodium dodecyl sulfate $5 \%$. The absorbance of the plates was read at $450 \mathrm{~nm}$ and the results were plotted using Prism 4.0 graphic package with non-linear analysis of regression ${ }^{51}$.

Reporting summary. Further information on research design is available in the Nature Research Reporting Summary linked to this article.

\section{Data availability}

The authors declare that the data that support the findings are included in the paper. Additional information is available from the corresponding author(s) upon request.

Received: 26 November 2018 Accepted: 23 July 2019

Published online: 13 August 2019

\section{References}

1. Kasturiratne, A. et al. The global burden of snakebite: a literature analysis and modelling based on regional estimates of envenoming and deaths. PLoS Med. 5, e218 (2008).

2. Scheske, L., Ruitenberg, J. \& Bissumbhar, B. Needs and availability of snake antivenoms: relevance and application of international guidelines. Int. J. Health Policy Manag. 4, 447-457 (2015).

3. Gutiérrez, J. M., León, G., Lomonte, B. \& Angulo, Y. Antivenoms for snakebite envenomings. Inflamm. Allergy Drug Targets 10, 369-380 (2011).

4. Fernández, J. et al. Venomic and antivenomic analyses of the Central American coral snake, Micrurus nigrocinctus (Elapidae). J. Proteome Res. 10, 1816-1827 (2011).

5. Laustsen, A. H., Lomonte, B., Lohse, B., Fernández, J. \& Gutiérrez, J. M. Unveiling the nature of black mamba (Dendroaspis polylepis) venom through venomics and antivenom immunoprofiling: Identification of key toxin targets for antivenom development. J. Proteom. 119, 126-142 (2015).

6. Sells, P. G., Jones, R. G. A., Laing, G. D., Smith, D. C. \& Theakston, R. D. G. Experimental evaluation of ovine antisera to Thai cobra (Naja kaouthia) venom and its $\alpha$-neurotoxin. Toxicon 32, 1657-1665 (1994).

7. Barber, C. M., Isbister, G. K. \& Hodgson, W. C. Alpha neurotoxins. Toxicon 66, 47-58 (2013)

8. Ranawaka, U. K., Lalloo, D. G. \& de Silva, H. J. Neurotoxicity in snakebitethe limits of our knowledge. PLoS Negl. Trop. Dis. 7, e2302 (2013).

9. Silva, A., Hodgson, W. C. \& Isbister, G. K. Antivenom for neuromuscular paralysis resulting from snake envenoming. Toxins (Basel) 9, 143 (2017).

10. de la Rosa, G., Corrales-García, L. L., Rodriguez-Ruiz, X., López-Vera, E. \& Corzo, G. Short-chain consensus alpha-neurotoxin: a synthetic 60-mer peptide with generic traits and enhanced immunogenic properties. Amino Acids 50, 885-895 (2018).

11. Laustsen, A. H., Lohse, B., Lomonte, B., Engmark, M. \& Gutiérrez, J. M. Selecting key toxins for focused development of elapid snake antivenoms and inhibitors guided by a Toxicity Score. Toxicon 104, 43-45 (2015).

12. Maeda, N. \& Tamiya, N. Three neurotoxins from the venom of a sea snake Astrotia stokesii, including two long-chain neurotoxic proteins with amidated C -termini. Biochem. J. 175, 507-517 (1978).

13. Engmark, M. et al. High-throughput immuno-profiling of mamba (Dendroaspis) venom toxin epitopes using high-density peptide microarrays. Sci. Rep. 6, 36629 (2016).

14. Chang, L.-S., Lin, J., Kuo, K.-W., Lin, S.-R. \& Chang, C.-C. Characterization of epitopes in native and unfolded cobrotoxin: evidence of an immunodominant C-terminal region related to the production of precipitating and nonprecipitating antibodies against cobrotoxin1. J. Biochem. 118, 686-692 (1995).

15. Menez, A. et al. Comparison of the "toxic" and antigenic regions in toxin a isolated from Naja nigricollis venom. Toxicon 20, 95-103 (1982).

16. Engmark, M., Jespersen, M. C., Lomonte, B., Lund, O. \& Laustsen, A. H. High density peptide microarray exploration of the antibody response in a rabbit immunized with a neurotoxic venom fraction. Toxicon 138, 151-158 (2017).

17. Lomonte, B. et al. Venoms of Micrurus coral snakes: evolutionary trends in compositional patterns emerging from proteomic analyses. Toxicon 122, 7-25 (2016).

18. Vergara, I. et al. Eastern coral snake Micrurus fulvius venom toxicity in mice is mainly determined by neurotoxic phospholipases $A_{2}$. J. Proteom. 105, 295-306 (2014).

19. de Roodt, A. R. et al. Effectiveness of two common antivenoms for North, Central, and South American Micrurus envenomations. J. Toxicol. Clin. Toxicol. 42, 171-178 (2004).

20. Olamendi-Portugal, T. et al. Proteomic analysis of the venom from the fish eating coral snake Micrurus surinamensis: novel toxins, their function and phylogeny. Proteomics 8, 1919-1932 (2008).

21. Tasoulis, T. \& Isbister, G. A review and database of snake venom proteomes Toxins (Basel). 9, 290 (2017).

22. Petras, D. et al. Snake venomics of African spitting cobras: toxin composition and assessment of congeneric cross-reactivity of the pan-African EchiTAbPlus-ICP antivenom by antivenomics and neutralization approaches. $J$. Proteome Res. 10, 1266-1280 (2011)

23. Calvete, J., Sanz, L., Pla, D., Lomonte, B. \& Gutiérrez, J. Omics meets biology: application to the design and preclinical assessment of antivenoms. Toxins (Basel). 6, 3388-3405 (2014).

24. Malih, I. et al. Proteomic analysis of Moroccan cobra Naja haje legionis venom using tandem mass spectrometry. J. Proteom. 96, 240-252 (2014).

25. Ramos-Cerrillo, B. et al. Characterization of a new polyvalent antivenom (Antivipmyn Africa) against African vipers and elapids. Toxicon 52, 881-888 (2008).

26. Lauridsen, L. P., Laustsen, A. H., Lomonte, B. \& Gutiérrez, J. M. Exploring the venom of the forest cobra snake: toxicovenomics and antivenom profiling of Naja melanoleuca. J. Proteom. 150, 98-108 (2017).

27. Dutta, S. et al. Proteomic analysis to unravel the complex venom proteome of eastern India Naja naja: correlation of venom composition with its biochemical and pharmacological properties. J. Proteom. 156, 29-39 (2017).

28. Arnberg, H., Eaker, D., Fryklund, L. \& Karlsson, E. Amino acid sequence of oxiana $\alpha$, the main neurotoxin of the venom of Naja naja oxiana. Biochim. Biophys. Acta 359, 222-232 (1974).

29. Kao, P.-H., Lin, S.-R. \& Chang, L.-S. Interaction of Naja naja atra cardiotoxin 3 with $\mathrm{H}$-trisaccharide modulates its hemolytic activity and membranedamaging activity. Toxicon 55, 1387-1395 (2010)

30. Kfir, R., Botes, D. P. \& Osthoff, G. Preparation and characterization of monoclonal antibody specific for naja nivea cardiotoxin VIII from the Naja and Hemachatus genera of the Elapidae family (SLOTTA and VICK, 1969; cardiotoxin peptide chain consists of 60-61 amino acids, cross-link. Toxicon 23, 135-144 (1985).

31. Shan, L.-L. et al. Proteomic characterization and comparison of venoms from two elapid snakes (Bungarus multicinctus and Naja atra) from China. J. Proteom. 138, 83-94 (2016).

32. Petras, D., Heiss, P., Harrison, R. A., Süssmuth, R. D. \& Calvete, J. J. Topdown venomics of the East African green mamba, Dendroaspis angusticeps, and the black mamba, Dendroaspis polylepis, highlight the complexity of their toxin arsenals. J. Proteom. 146, 148-164 (2016).

33. Mourier, G. et al. Mambalgin-1 pain-relieving peptide, stepwise solid-phase synthesis, crystal structure, and functional domain for acid-sensing ion channel 1a inhibition. J. Biol. Chem. 291, 2616-2629 (2016).

34. Fruchart-Gaillard, C. et al. Engineering of three-finger fold toxins creates ligands with original pharmacological profiles for muscarinic and adrenergic receptors. PLoS ONE 7, e39166 (2012). 
35. Oyama, E. \& Takahashi, H. Purification and characterization of two plateletaggregation inhibitors, named angustatin and $\mathrm{H}$-toxin $\mathrm{TA} 2$, from the venom of Dendroaspis angusticeps. Toxicon 93, 61-67 (2015).

36. Blanchet, G. et al. Polypharmacology profiles and phylogenetic analysis of three-finger toxins from mamba venom: case of aminergic toxins. Biochimie 103, 109-117 (2014).

37. Servent, D. et al. Muscarinic toxins. Toxicon 58, 455-463 (2011).

38. Silva, A., Cristofori-Armstrong, B., Rash, L. D., Hodgson, W. C. \& Isbister, G. $\mathrm{K}$. Defining the role of post-synaptic a-neurotoxins in paralysis due to snake envenoming in humans. Cell. Mol. Life Sci. 75, 4465-4478 (2018).

39. Vonk, F. J. et al. The king cobra genome reveals dynamic gene evolution and adaptation in the snake venom system. Proc. Natl Acad. Sci. USA 110, 20651-20656 (2013).

40. Danpaiboon, W. et al. Ophiophagus hannah venom: proteome, components bound by Naja kaouthia antivenin and neutralization by N. kaouthia neurotoxin-specific human ScFv. Toxins (Basel). 6, 1526-1558 (2014).

41. de la Rosa, G., Pastor, N., Alagón, A. \& Corzo, G. Synthetic peptide antigens derived from long-chain alpha-neurotoxins: immunogenicity effect against elapid venoms. Peptides 88, 80-86 (2017).

42. Gowtham, Y. J., Mahadeswaraswamy, Y. H., Girish, K. S. \& K, K. Crossreactivity and neutralization of Indian King cobra (Ophiophagus hannah) venom by polyvalent and monovalent antivenoms. Int. Immunopharmacol. 21, 148-155 (2014).

43. Ismail, M., Abd-Elsalam, M. A. \& Al-Ahaidib, M. S. Pharmacokinetics of ${ }^{125}$ Ilabelled Walterinnesia aegyptia venom and its specific antivenins: flash absorption and distribution of the venom and its toxin versus slow absorption and distribution of $\operatorname{IgG}, \mathrm{F}\left(\mathrm{ab}^{\prime}\right) 2$ and $\mathrm{F}(\mathrm{ab})$ of the antivenin. Toxicon 36, 93-114 (1998).

44. Ismail, M., Al-Ahaidib, M. S., Abdoon, N. \& Abd-Elsalam, M. A. Preparation of a novel antivenom against Atractaspis and Walterinnesia venoms. Toxicon 49, 8-18 (2007).

45. Lomonte, B. et al. Two color morphs of the pelagic yellow-bellied sea snake, Pelamis platura, from different locations of Costa Rica: snake venomics, toxicity, and neutralization by antivenom. J. Proteom. 103, 137-152 (2014).

46. Silva, A., Hodgson, W. \& Isbister, G. Cross-neutralisation of in vitro neurotoxicity of asian and australian snake neurotoxins and venoms by different antivenoms. Toxins (Basel). 8, 302 (2016).

47. Chippaux, J.-P., Williams, V. \& White, J. Snake venom variability: methods of study, results and interpretation. Toxicon 29, 1279-1303 (1991).

48. de Roodt, A. R., Lanari, L. C., Costa de Oliveira, V., Laskowicz, R. D. \& Stock, R. P. Neutralization of Bothrops alternatus regional venom pools and individual venoms by antivenom: a systematic comparison. Toxicon $\mathbf{5 7}$ 1073-1080 (2011).

49. Clement, H. et al. Heterologous expression, protein folding and antibody recognition of a neurotoxin from the Mexican coral snake Micrurus laticorallis. J. Venom. Anim. Toxins Incl. Trop. Dis. 22, 25 (2016).

50. Guerrero-Garzón, J. F. et al. Cloning and sequencing of three-finger toxins from the venom glands of four Micrurus species from Mexico and heterologous expression of an alpha-neurotoxin from Micrurus diastema. Biochimie 147, 114-121 (2018).

51. de la Rosa, G., Olvera, F., Cruz, E., Paniagua, D. \& Corzo, G. Use of irradiated elapid and viperid venoms for antivenom production in small and large animals. Toxicon 155, 32-37 (2018).

52. Rojas, G., Jiménez, J. \& Gutiérrez, J. Caprylic acid fractionation of hyperimmune horse plasma: description of a simple procedure for antivenom production. Toxicon 32, 351-363 (1994).
53. Chippaux, J.-P. Guidelines for the production, control and regulation of snake antivenom immunoglobulins. Biol. Aujourd'hui. 204, 87-91 (2010).

54. WHO. Progress in the characterization of venoms and standardization of antivenoms. WHO offset Publ. https://doi.org/10.1016/0041-0101(81)90095-7, $1-44$ (1981).

\section{Acknowledgements}

We acknowledge Ricardo Mondragon, M. en C. Alejandro Olvera, M. en Biotec. Herlinda Clement, Dr. Bradley Yates, and personal from "Ojo de Agua" Ranch and Instituto Clodomiro Picado for providing technical support. This work received funding from the Direccion General de Asuntos del Personal Academico (DGAPA-UNAM) grant number IN203118 awarded to G.C. and IN207218 awarded to A.A. Partial financial support by Vicerrectoria de investigacion, Universidad de Costa Rica (VI-B7608), awarded to B.L. is gratefully acknowledged.

\section{Author contributions}

G.d.l.R. performed Lot $\# 1$ and the other experiments and wrote the manuscript. I.G.A. performed the second Lot $\# 2$ of experiments. F.O. performed the purification of IgGs. A.A. and B.L. contributed to the study concept, design, and sample acquisition. G.d.l.R. and G.C. conceived and designed the project. G.C. served as principal investigator. All authors discussed the results, contributed to critical revisions, and approved the final manuscript.

\section{Additional information}

Supplementary Information accompanies this paper at https://doi.org/10.1038/s41467 019-11639-2.

Competing interests: The authors declare no competing interests.

Reprints and permission information is available online at http://npg.nature.com/ reprintsandpermissions/

Peer review information: Nature Communications would like to thank Andreas Laustsen and other, anonymous, reviewers for their contributions to the peer review of this work. Peer reviews reports are available.

Publisher's note: Springer Nature remains neutral with regard to jurisdictional claims in published maps and institutional affiliations.

Open Access This article is licensed under a Creative Commons Attribution 4.0 International License, which permits use, sharing, adaptation, distribution and reproduction in any medium or format, as long as you give appropriate credit to the original author(s) and the source, provide a link to the Creative Commons license, and indicate if changes were made. The images or other third party material in this article are included in the article's Creative Commons license, unless indicated otherwise in a credit line to the material. If material is not included in the article's Creative Commons license and your intended use is not permitted by statutory regulation or exceeds the permitted use, you will need to obtain permission directly from the copyright holder. To view a copy of this license, visit http://creativecommons.org/ licenses/by/4.0/.

(C) The Author(s) 2019 